It seems probable that both classes of effects are involved in the actual magnetisation in question, though experiment has shown that any effect of class $(a)$ is at least exceedingly minute unless the magnetic behaviour of the interior parts of the earth and sun is quite different from that of matter at ordinary temperatures on the surface of the earth.

The Ohio State University, Columbus, S. J. BarnetT. Ohio, U.S.A., March 12.

\section{A Triangle that gives the Area and Circumference of any Circle, and the Diameter of a Circle equal in Area to any given Square.}

IT is not possible to measure exactly the interminable fraction required for the line $\mathrm{BZ}$ in the following figure, but it is quite easy to draw it so nearly that the error is practically immensurable.

First Method.-Draw a line $\mathrm{AB}=44$, and make $\mathrm{BZ}=23 .{ }_{44}^{23}=0.5227^{2} \overline{7}^{2}$, which is a little too long.

AY will then be short, about $I$ in 600,000 .

AX will also be short, about $I$ in 300,000 .

Second Method (with the familiar ratio $\frac{355}{113}$ ). Make a circle with diameter $\mathrm{AB}=\mathrm{II} \cdot 3$, and let $\mathrm{AX}=8 \frac{7}{8}$. Draw a perpendicular from $X$ to cut the circle in $Y$. Join $\mathrm{AY}$, and continue the line to $\mathrm{Z}$.

Then the error in $\mathrm{AX}$, the $\frac{1}{4}$ circumference, will be less than $I$ in $I I, 780,000$ in excess.

The true angle for the line $\mathrm{AZ}$ lies between the lines found by the two methods, but the difference is too small for measurement, and in any accurate drawing the lines will appear to coincide.

$\mathrm{AX}$ being found, equal to an arc of $90^{\circ}$, a line for any other arc may be found; and the triangle once drawn on a sufficiently large scale, is true for all circles.

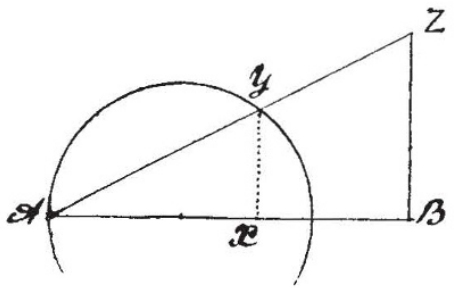

Let $A B=I$, and at right angles

$\mathrm{BZ}=0^{\circ} 5227232008+$, join AZ $\}$ Angle A. $27^{\circ} 35^{\prime} 49^{\circ} 6^{\prime \prime}+$

Then, any circle with diameter upon $\mathrm{AB}$ and one extremity at $A$, will cut the line $A Z$ (or $A Z$ produced) in a point $\mathrm{Y}$, making $\mathrm{AY}$ the side of a square equal in area to the circle.

Also, a line from $\mathrm{Y}$ perpendicular to $\mathrm{AB}$ will cut the diameter in a point $\mathrm{X}$, making $\mathrm{AX}$ equal to $\frac{1}{4}$ circumference of the circle.

Again, any square with base upon $\mathrm{AB}$ and a corner at $\mathrm{A}$, will, with its side opposite to $\mathrm{A}$, cut $\mathrm{AZ}$ in a point $\mathrm{Y}$, making $\mathrm{AY}$ the diameter of an equal circle.

\section{The Croft, Tenby.} T. M. P. Hughes.

THE following remarks may help to explain $\mathrm{Mr}$. Hughes's constructions :-

Let $A Y$ be a chord of a circle of which $A D$ is a diameter, and let $\angle D A Y=\theta$. Then if the square on $\mathrm{AY}$ equals the area of the circle, $(2 r \cos \theta)^{2}=\pi r^{2}$, and therefore $\cos ^{2} \theta=\pi / 4, \tan ^{2} \theta=(4-\pi) / \pi$, and

$$
\tan \theta=0.5^{227232}
$$

very nearly, as stated. Now if we express this NO. 23 I8, VOL. 93] approximate value of $\tan \theta$ as an ordinary continued fraction we find the successive convergents,

$$
\text { I/I, I/2, II } / 2 \text { I, I2 } / 23,23 / 44 \text {, etc., }
$$

where the first of those not written has four digits in numerator and denominator. Hence, as Mr. Hughes has discovered, $23 / 44$ is a very close approximation to the transcendent number $\sqrt{ }(4-\pi) / \sqrt{ } \pi$. It seems absurd to speak of a mathematical accident, but we do seem to have something of the kind here. Supposing that $a / b$ is a rational approximation to $\sqrt{ }(4-\pi) / \sqrt{ } \pi$, we should not expect beforehand a solution correct within about $4 \cdot 10^{-6}$ for values of $a, b$, each less than Ioo.

The second construction is obtained by putting, as an approximation,

$\mathrm{AX}: \mathrm{AD}=\pi / 4=355 / 4 \cdot \mathrm{I} \mathrm{I} 3=7 \mathrm{I0} / 8 \cdot \mathrm{II}_{3}=8 \frac{7}{8} / \mathrm{II} \cdot 3$.

It would be easy to make a set-square with its shorter sides in the ratio $23: 44$, and this could be used for the approximate quadrature and rectification of any given circle. It is interesting to see how the same figure solves both problems to the same degree of exactness (practically). I suppose the error in the set-square could be reduced to $\mathrm{O}$.I per cent., or less; the question is, what percentage of error is likely to occur in using it. For the rectification we have to draw the perpendicular YX; it seems to me that for the quadrature we are likely to obtain the most accurate results by using a straight edge as well as the set-square; that is to say, we should not try to adjust the set-square without first placing a straight edge along a diameter of the circle. If this is so, the graphical solutions of both problems are likely to be affected by the same percentage of error; because to obtain $\mathrm{X}$, after marking $\mathrm{Y}$, we have only to slide the set-square along the straight edge until a shorter side goes through $\mathrm{Y}$; and if we repeat the manipulation several times, I do not think the error in finding $\mathrm{X}$, regarded as a distance from the true position, can be so much as five times the error in finding $\mathrm{Y}$, or conversely. Of course, by "the same percentage of error" I mean here that the two errors, on the same scale, are of the orders $\pm a \cdot 10^{-n}, \pm b \cdot 10^{-n}$, where $a, b$ both lie above $\mathrm{I}$, while neither of them is equal to, or exceeds, 5 .

G. B. Mathews.

\section{New Units in Aerology.}

With reference to Prof. McAdie's letter in Nature of March I9, p. 58, I should like to point out that throughout my "Thermodynamics," published in 1878 , a megadyne per square centimetre is used as the unit of pressure, and it is termed a c.g.s. atmosphere. Ever since $\mathrm{x} 888$, when the B.A. committee (of which I was a member), adopted the barad, I have employed in my lectures the above pressure unit under the name of megabarad. The corresponding unit of work, and also of heat, adopted in the book is the megalerg. Megerg to my ear is too cacophonous for use.

Christ Church, Oxford, March 25. ROBERT E. BAYNES.

\section{PROGRESS IN WIRELESS TELEPHONY.}

THE attention of telephonic engineers has of late years been very closely directed to the improvement of the line wire in ordinary telephony. Apart from the imperfections of the telephone transmitter and receiver, per se, a very considerable effect is produced on the transmitted speech by the line itself if it is at all long. This action, from an electrical point of view, consists in the distortion of the wave form of the current 